\title{
The relationship between caregiver impacts and the unmet needs of survivors of stroke
}

\author{
This article was published in the following Dove Press journal: \\ Patient Preference and Adherence \\ 27 July 2015 \\ Number of times this article has been viewed
}

\author{
Nadine E Andrew' \\ Monique F Kilkenny ${ }^{1,2}$ \\ Rebecca Naylor ${ }^{3}$ \\ Tara Purvis' \\ Dominique A Cadilhac ${ }^{1,2}$ \\ 'Translational Public Health and \\ Evaluation Division, Stroke and \\ Ageing Research, School of Clinical \\ Sciences at Monash Health, Monash \\ University, Clayton, ${ }^{2}$ Florey Institute \\ of Neuroscience and Mental Health, \\ Heidelberg, ${ }^{3}$ National Stroke \\ Foundation, Melbourne, VIC, Australia
}

Background: Caregivers play a crucial role in meeting the needs of survivors of stroke. Yet, little is known about how they are impacted by their caregiving role.

Objectives: To describe the relationship between survivor long-term unmet needs ( $>12$ months) and caregiver impacts, and identify characteristics that are associated with reported moderate to severe impacts on caregivers.

Method: This was a cross-sectional survey using data from the Australian Stroke Survivor and Carer Needs Survey. Community dwelling adults $12+$ months poststroke and their caregivers participated. Caregivers and survivors were asked about the extent to which the domains of work, leisure and family, and friend and spousal relationships had been impacted using a Likert scale of responses. The extent to which survivor needs were being met was measured over the domains of health, everyday living, work, leisure, and finances, and the total number of unmet needs was calculated. The association between survivor unmet needs and caregiver impacts was assessed using multivariable logistic regression adjusted for caregiver and survivor characteristics.

Results: Of the 738 completed survivor surveys, 369 contained matched caregiver data (survivors: median age, 71 years; 67\% male) (caregivers: median age, 64 years; 26\% male). For caregivers, the domains of work, leisure, and friendships were most impacted. The odds of a caregiver experiencing moderate to extreme impacts increased with the number of reported survivor unmet needs. This was greatest for spousal (aOR [adjusted odds ratio]: 1.14; 95\% CI [confidence interval]: 1.07, 1.21; $P<0.001$ ) and friend relationships (aOR: 1.14; 95\% CI: 1.07, $1.21 ; P<0.001)$. Caring for a survivor who needed daily living assistance was associated with moderate to extreme caregiver impacts across all domains.

Conclusion: Caregivers of survivors of stroke experience large negative impacts, the extent to which is associated with survivors unmet needs. Targeted, long-term solutions are needed to support survivors and caregivers living in the community.

Keywords: outcomes, community care, disability, burden, support

\section{Introduction}

Stroke is the second leading cause of adult disability in developed countries. In Australia, the majority of survivors of stroke (up to 90\%) live at home and most are dependent on others to help them with everyday activities. ${ }^{1}$ There are limited formal long-term support services available for survivors living in Australia and other developed countries, especially beyond the first 6-12 months following stroke. Once back living in the community, survivors of stroke predominantly rely on their general medical practitioner as their primary point of care contact. ${ }^{2}$ Availability of support services vary between different geographic areas, and stroke-specific services and rehabilitation are often difficult to access on an ongoing basis, especially for those who cannot afford
Correspondence: Nadine E Andrew Translational Public Health and Evaluation Division, Stroke and Aging Research, School of Clinical Sciences at Monash Health, Monash University, Clayton, VIC 3800 , Australia

Tel +6I 395947509

Fax +6I 399024245

Email nadine.andrew@monash.edu 
to access private services. Consequently, the majority of the care burden often falls upon family members.

Support from family or informal caregivers (ie, those who provide day-to-day care for survivors of stroke) is important in meeting the needs of the majority of survivors of stroke, and frequently involves the provision of practical help such as assistance with activities of daily living (ADLs) and emotional support. ${ }^{3,4}$ The use of informal support such as family and friends has also been reported as an invaluable and key component in facilitating recovery following stroke. ${ }^{5,6}$ However, this can be at a significant personal and financial cost to caregivers. For example, caregiver time costs have been estimated to be between $14 \%$ and $23 \%$ of the lifetime cost of a first ever stroke or AUS\$42.5 million (replacement cost approach), with most of this care $(>90 \%)$ provided during caregivers leisure time. ${ }^{7}$ Primary caregivers have also been reported to spend a median of 17-32 hours a week performing caregiver-related tasks, such as assistance with self-care, driving the survivor to appointments or other community-based activities, or performing domestic duties previously performed by the survivor. ${ }^{7,8}$ Personal costs or consequences frequently experienced by caregivers include impacts on quality of life $e^{8,9}$ as well as increased frequency of anxiety and depression compared to those not in caregiver roles. ${ }^{10,11}$ For the caregiver, this can also have detrimental effects on relationships both inside and outside the family and their ability to participate in work and leisure activities. ${ }^{12}$

In Australia, caregivers of survivors of stroke report having many needs that are not being adequately met, especially with regard to their social lives, tiredness, and their own distress at the stroke survivor's condition. ${ }^{13}$ Many have reported dissatisfaction with access to services and the quality of the services that are available. ${ }^{12}$ In the early period following stroke, many caregivers have expressed feeling unsupported and worried about the uncertainty of their role. ${ }^{6,14,15}$ This is concerning given that low levels of social support have been shown to be associated with caregiver anxiety, depression, and irritability. ${ }^{11}$

There is consensus from governments, consumers, and researchers that more needs to be done to support caregivers. ${ }^{16}$ However, to date, there are no studies that have investigated the influence of survivor unmet needs on caregivers. The aims of our study were to describe the association between survivor long-term unmet needs, 12 or more months following stroke, and caregiver impacts; and identify survivor and caregiver characteristics that are associated with reported moderate to extreme impacts on caregivers' lives.

\section{Methods}

\section{Survey design and development}

Data from the Australian Stroke Survivor and Carer Needs Assessment Project (2012), commissioned by the National Stroke Foundation (NSF), were used. The survey contained a survivor component and an optional caregiver component. Detailed methods for this study and the results from the survivor component are reported elsewhere. ${ }^{17}$ In brief, the survivor component was an Australian adaptation of the UK Stroke Survivors Needs Survey. ${ }^{18}$ The Australian version contained additional questions relating to survivor unmet needs across the domains of health, everyday living, work, leisure, social support, and finances. Many of the questions in the unmet health needs domain were consistent with the UK survey. The caregiver component was developed specifically for this Australian-based project. Input for both components was provided from an expert advisory group established and chaired by the NSF that consisted of representatives from Monash University, members of the United Kingdom research team, general practitioners, stroke researchers, and consumers. The expert group provided consensus with regard to items considered to be important to long-term survivors of stroke and their caregivers, and offered feedback during the pretesting phase of the survey development. The survey was pilot tested in a sample of survivors of stroke $(n=15)$ and their caregivers. In the final survey $(n=765), 88 \%$ of respondents felt that their needs were adequately covered by the survey, indicating good content validity.

In the survey, both survivors and caregivers were asked about the extent to which their lives had been impacted, since having a stroke or taking on a caregiver role, across the domains of work, leisure, family relationships, spousal relationships, and friend relationships, using a 5-point Likert scale of responses. The caregivers component contained demographic questions and questions about the caregivers relationship to the survivor, their living arrangements, previous caregiver experience, and time in their caregiver role. Caregivers were also asked about whether or not their support and care needs were being met, the financial impacts of taking on a caregiver role, and their experiences using community health services and respite care. Open-ended questions were provided at the end of each section to allow participants to provide additional information. A copy of the survey can be obtained by emailing the corresponding author.

\section{Recruitment and data collection}

Participant recruitment occurred between December 2011 and December 2012. Eligibility criteria for survivor 
participants include the following: being aged 18 years or over, living in the community, having a clinical diagnosis of stroke, having had their first stroke at least 1 year prior to survey completion, and being able to complete a survey (with or without support from a friend, caregiver, or family member) within the project timeframe. Eligible caregivers were any adults providing day-to-day care for the survivor participant. Surveys could be either paper-based (85\%), online $(25 \%)$, or telephonic $(<1 \%)$. Paper-based surveys were provided with two reply paid envelopes, one for the survivor survey and one for the caregiver survey, so the caregiver survey could remain confidential if desired. Results from the two surveys were merged using a common survey identification number.

A two-phased, multifaceted, cross-sectional sampling strategy was used to recruit a broad sample of survivors of stroke and their caregivers. In the first phase, readily available recruitment sources were utilized. These included 1) the Australian Stroke Clinical Registry (AuSCR - www.auscr.com.au), a collaborative national registry with the aim to monitor, promote, and improve the quality of acute stroke care; 2) NSF Stroke Connect members, Stroke Connect online members, and stroke support group members (www.strokefoundation.com.au/afterstroke/ get-help), these are support programs for survivors of stroke, caregivers, friends, and family; and 3) the NSF website which provided a link to an online version of the survey.

Following completion of the first phase, an interim analysis was performed to identify coverage gaps based on the area of residence. Based on this analysis, hospitals were purposefully selected to ensure representation from all Australian states and an appropriate mix of rural/urban coverage. Nonresponder data were available for those recruited through the AuSCR and were used to establish the generalizability of our results.

\section{Ethics}

The project was approved by the Monash University Human Research Ethics Committee (HREC) (Approval number CF11/2579 - 2011001510). Individual ethics approval was also obtained from the HREC, responsible for each of the hospitals that participated in the project $(n=18)$. Approval was also granted by the AuSCR Research Task Group and Management Committee.

\section{Definitions}

For the survivor needs assessment component of the survey, responses to the question "have you had enough help with..." were 1) yes definitely, 2) to some extent, 3) no, I did not get enough help, 4) I did not want help, or 5) I did not have difficulties with. A need was defined as "not fully met" if the response to the question "have you had enough help with..." was 1) "yes, to some extent" or 2) "no, I did not get enough help". The response "I did not have difficulties with" was used to determine whether or not the survivor had problems in the area being measured, for example, mobility or emotional problems. When calculating the proportions of survivors with unmet needs only those with problems in the area being described were included in the denominator. The total number of unmet needs was calculated by summing the number of needs reported as not fully met across each of the domains. A survivor was considered to need assistance with ADLs if they answered yes to the question "do you currently need help from anybody with everyday activities". Whether or not the survivor needed help with ADLs was used to define the level of disability for the subgroup analyses. Cognitive and emotional needs encompassed nonphysical health needs such as concentration, memory, cognition, fatigue, and emotions. Table 1 shows the subcategories included in each of the needs domains. Work was defined as vocational work or paid employment.

To determine the level of impact that stroke had on survivors and caregivers, questions for each domain such as "have your hours of work (or study) changed..." were categorized as 1) not at all, 2) a little, 3) moderately, 4) a lot, 5) extreme, or 6) cannot answer. To allow for sufficient numbers for multivariable analyses, the data analyses responses were dichotomized so that "moderate", "a lot", and "extreme" were combined to indicate "moderate to extreme" impacts and "not at all" or "a little" indicated "little or no" impacts. The response "cannot answer" was excluded from the denominator.

\section{Data analyses}

Only matched samples of survivor and caregiver data from the survey were used. Descriptive statistics were used to describe the proportion of caregivers and survivors who reported moderate to extreme impacts. Univariable logistic regression was used to assess the association between caregiver impacts and survivor impacts. Where data were missing for a question, these data were excluded from the analysis ( $\sim 4 \%-6 \%$ of responses).

Multivariable logistic regression (model 1) was used to assess association between the number of long-term unmet needs reported by the survivor and whether or not the caregiver experienced moderate to extreme impacts. Models were adjusted for survivor disability (difficulties with ADLs), health and disability problems reported by the survivor, time since stroke, whether or not the survivor 


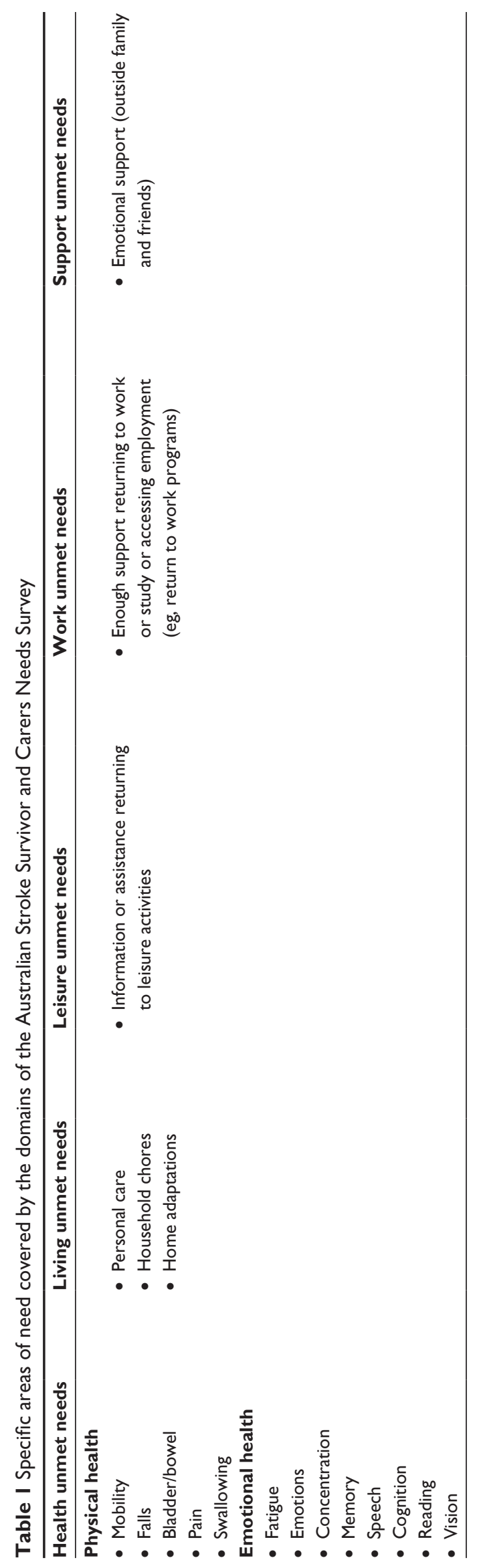

was living with the caregiver, caregiver age, caregiver sex, survivor age, survivor sex, and whether or not the caregiver was previously a caregiver.

Multivariable logistic regression (model 2) was also used to assess for factors associated with moderate to extreme impacts on caregivers' lives across the domains of leisure, work, spousal relationships, family relationships, and friend relationships. Independent variables were selected based on a univariable statistical significance of $P<0.1$ to allow for the effect of both negative and positive associations within the model. Where large numbers of variables were associated with the outcome, backward stepwise regression was used to obtain the final models. A Bonferroni adjustment of the $P$-value was also calculated for the multivariable analyses, and both adjusted and unadjusted results were reported. All models were tested for collinearity, and a condition index of $<30$ was considered acceptable. ${ }^{19}$ Stata (version 12 , StataCorp LP, College Station, TX, USA, 2013) statistical software was used, and a $P$-value of $<0.05$ was considered to be statistically significant.

\section{Results}

A total of 765 survivors completed the survivor survey, of these 369 (48\%) surveys contained matched survivor and caregiver responses and were included in the analyses. Most responses (172, 47\%) were obtained from the hospital recruitment strategy, followed by 87 (24\%) from AuSCR, 62 (17\%) from NSF program members, and 48 (13\%) from other sources. Respondents were from all states and territories in Australia and from both rural and urban settings. For survivors recruited through AuSCR, responders were similar to nonresponders, except that responders were more likely to be male. ${ }^{17}$

Characteristics of caregivers, survivors with matched caregiver responses, and all survivor respondents are shown in Table 2. Compared to caregivers, survivors were more likely to be male (survivors: $67 \%$ male, $33 \%$ female vs caregivers: $26 \%$ male, $74 \%$ female), and caregivers were approximately 7 years younger than survivors. The majority of caregivers were relatives of the survivor (Table 2). Survivor Needs Survey participants with caregiver respondents were significantly older, were more likely to be male, and were more likely to require assistance with ADLs. In Figure 1, the most common needs reported by survivors as not being fully met are shown. The median number of unmet needs reported by survivors was $6(\mathrm{Q} 1, \mathrm{Q} 3: 2,10)$.

Although survivors were more likely to report moderate to severe impacts than their caregivers, the caregivers were also impacted greatly. Among those working prior to 
Table 2 Characteristics of caregivers, survivors with matched caregiver data, and all survivor respondents

\begin{tabular}{|c|c|c|c|}
\hline & $\begin{array}{l}\text { Survivors with caregiver data } \\
\mathbf{N}=369^{a} \\
\text { n (\%) }\end{array}$ & $\begin{array}{l}\text { Caregivers } \\
\mathrm{N}=\mathbf{3 6 9} \\
\mathrm{n}(\%)\end{array}$ & $\begin{array}{l}\text { All survivor respondents } \\
\mathrm{N}=765^{\mathrm{c}} \\
\mathrm{n}(\%)\end{array}$ \\
\hline Age (median: QI, Q2) & $71(62,80)$ & $64(54,72)$ & $68(59,77)^{\mathrm{b}}$ \\
\hline Sex (male) & $246(67)$ & $94(26)$ & $445(58)^{\mathrm{b}}$ \\
\hline \multicolumn{4}{|l|}{ Location $^{\mathrm{a}}$} \\
\hline Major city & $208(60)$ & & $417(62)$ \\
\hline Inner regional & $93(27)$ & & $173(26)$ \\
\hline Outer regional & $47(14)$ & & $87(13)$ \\
\hline Needs assistance with ADLs & $208(57)$ & & $280(40)^{b}$ \\
\hline Years since stroke (median: Q1, Q2) & $I(I, 2)$ & & $2(2,4)$ \\
\hline Years in caregiver role & & $2(2,5)$ & \\
\hline Lives with survivor & & $321(88)$ & \\
\hline Previous caregiver experience & & $52(15)$ & \\
\hline
\end{tabular}

Notes: ${ }^{\mathrm{a}} \mathrm{n}=2 \mathrm{I}(6 \%)$ had missing location data; ${ }^{\mathrm{b}} \mathrm{P}<0.05$ difference between survivors with and without caregiver data; ${ }^{\mathrm{n}} \mathrm{n}=88(\mathrm{I} \%)$ had missing location data.

Abbreviations: ADLs, activities of daily living; QI, quartile I; Q2, quartile 2.

stroke, $40 \%$ of caregivers and $67 \%$ of survivors reported a moderate to extreme reduction in the amount of vocational work that they were able to perform. Almost half of the caregivers $(47 \%)$ and $70 \%$ of survivors reported a moderate to extreme reduction in the number or type of leisure activities that they were able to undertake (Figure 2). Significant interrelationships were demonstrated between survivor and caregiver impacts across all domains, especially the relationship domains (Table 3). For example, if the survivors reported moderate to extreme impacts on their spousal relationship, the odds of the caregiver also reporting moderate to extreme impacts were approximately 17 times more than for survivors who did not report moderate to extreme impacts.

Almost one-quarter of caregivers (21\%) reported that they were not receiving enough social support in their caregiver role. Almost one in three reported experiencing a loss of income (28\%) and half (50\%) reported experiencing an increase in personal expenses since taking on a caregiver role. Over half (54\%) of the caregivers reported that they needed

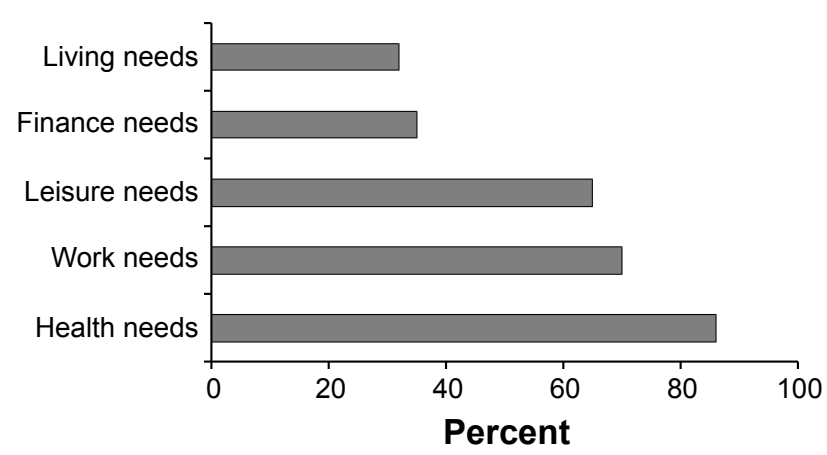

Figure I Proportion of survivors of stroke with needs not fully met for each domain measured. to use respite care. However, of those needing respite care $24 \%$ were unable to access it.

Results from our multivariable analyses (model 1) showed that caregiver impacts across all domains except work were significantly associated with the total number of unmet needs reported by survivors (Table 4). This was greatest for spousal relationships and friend relationships, so that for every additional unmet need reported by the survivor there was a $14 \%$ increase in the odds of the caregiver reporting moderate to extreme impacts in these domains.

Our multivariable analyses (model 2) showed that caring for a survivor who needed assistance with ADLs was most strongly associated with moderate to extreme impacts on caregivers across all domains, especially work and leisure (Table 5). Caregivers of survivors with cognitive problems had a three times greater odds of experiencing moderate to extreme impacts on spousal relationships, and caregivers who had previously been in a caregiver role had a six times greater odds of experiencing moderate to extreme impacts on

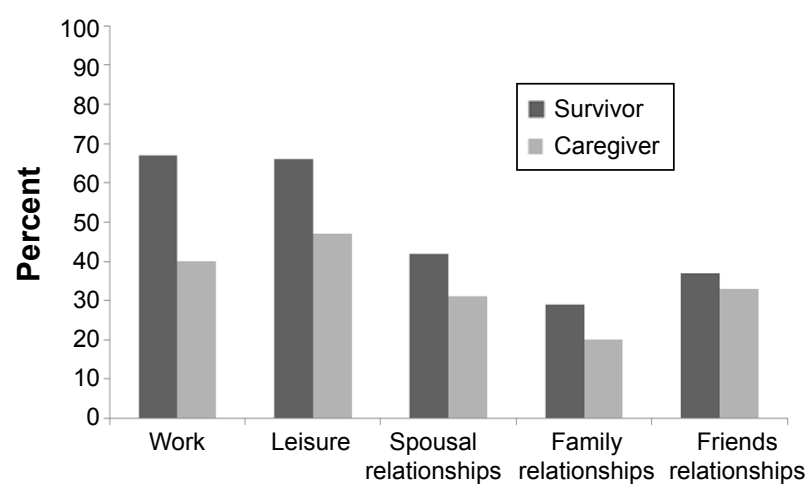

Figure 2 Proportion of survivors and caregivers reporting moderate to extreme changes for each domain measured. 
Table 3 Univariable logistic regression results showing the association between survivor and caregiver impacts across the domains measured in the survey

\begin{tabular}{lllll}
\hline Domain & $\mathbf{n}$ & OR & $\mathbf{9 5 \% ~ C l ~}$ & P-value \\
\hline Work $^{\mathrm{a}}$ & 156 & 8.2 & $3.5,19.2$ & $<0.00 \mathrm{I}$ \\
Leisure $^{\mathrm{a}}$ & 324 & 9.0 & $5.0,16.1$ & $<0.00 \mathrm{I}$ \\
Spousal relationships $^{\mathrm{b}}$ & 293 & 17.5 & $9.2,33.0$ & $<0.00 \mathrm{I}$ \\
Family relationships & 339 & 11.5 & $6.2,21.4$ & $<0.00 \mathrm{I}$ \\
Friend relationships & 346 & 12.3 & $7.2,21.0$ & $<0.00 \mathrm{I}$ \\
\hline
\end{tabular}

Notes: ${ }^{a}$ Only those who participated in these activities prior to stroke were included; bonly those with a spouse were included.

Abbreviations: $\mathrm{OR}$, odds ratio; $\mathrm{Cl}$, confidence interval.

family relationships. Being a younger caregiver $(<65$ years $)$ was associated with needing more support (Table 5).

\section{Discussion}

To our knowledge, this is the first large survey in which the association between survivor unmet needs and caregiver impacts has been investigated. Our results provide evidence that the impacts on caregivers of survivors of stroke are substantial and that these impacts are positively associated with survivor impacts and survivor unmet needs. The extent to which a caregiver was impacted was most strongly influenced by survivor characteristics, especially disability level (ie, if the survivors needed assistance with ADLs), cognitive problems, and emotional problems. These data are important for informing strategies to support caregivers of survivors of stroke.

Meeting the long-term unmet needs of survivors of stroke may help reduce caregiver impacts. Our results provide evidence that when survivor needs were not fully met, caregivers experienced greater impacts, and that caregiver impacts were predominantly influenced by survivor characteristics. This is consistent with other literature that has shown increased

Table 4 Multivariable results (model I): association between reported number of survivor unmet needs and caregiver impacts across the five domains

\begin{tabular}{llll}
\hline Domains impacted & \multicolumn{3}{l}{ Number of survivor unmet needs } \\
\cline { 2 - 4 } & aOR & $\mathbf{9 5 \%} \mathbf{~ C l}$ & P-value \\
\hline Work & 1.08 & $1.00,1.17$ & 0.06 \\
Leisure & 1.10 & $1.03,1.17$ & 0.004 \\
Spousal relationship & 1.14 & $1.07,1.21$ & $<0.001$ \\
Family relationship & 1.10 & $1.03,1.18$ & 0.008 \\
Friend relationship & 1.14 & $1.07,1.21$ & $<0.00 \mathrm{I}$ \\
\hline
\end{tabular}

Notes: Models adjusted for survivor disability (difficulties with activities of daily living), survivor having problems, time since stroke, whether or not the survivor was living with the caregiver, caregiver age, caregiver sex, survivor age, survivor sex, and whether or not the caregiver was previously a caregiver.

Abbreviations: aOR, adjusted odds ratio; $\mathrm{Cl}$, confidence Interval.

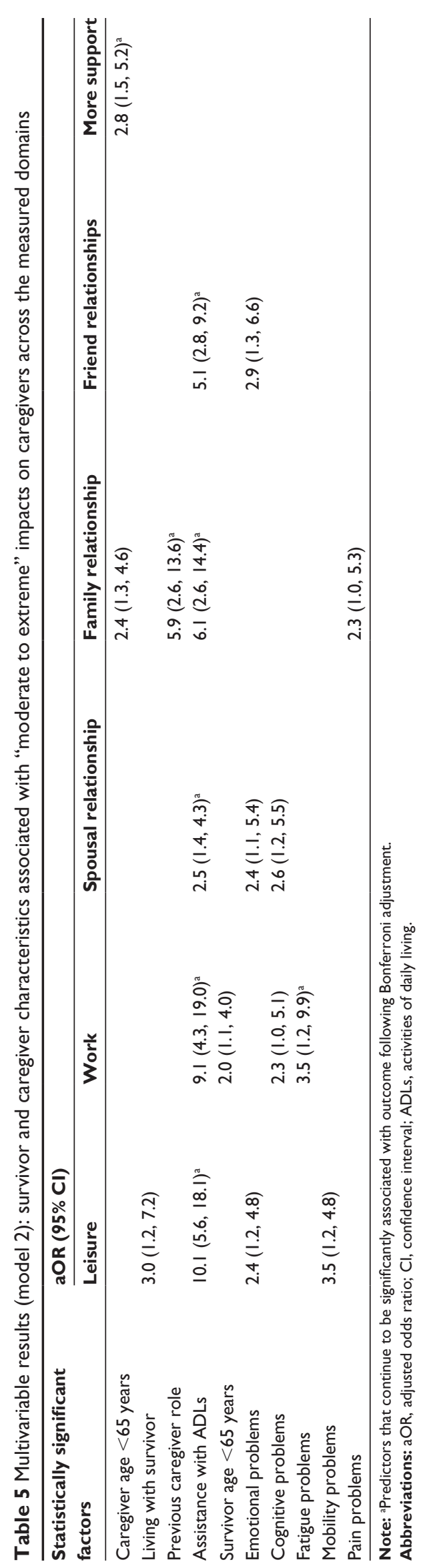


caregiver burden and reduced quality of life in those caring for survivors with greater levels of physical disability or with cognitive or emotional problems. ${ }^{8,11,20-22}$ When survivor needs are not met by health services, additional demands are placed on the caregiver, which can lead to feelings of inadequacy, frustration, and disempowerment when they are unable to meet the survivor's needs. Further, caregiver needs have been shown to be strongly tied to the survivors' ability to access appropriate services and health professional support. ${ }^{12,23}$ In particular, more needs to be done to address the nonphysical consequences of stroke, as highlighted in the main results from the Australian Stroke Survivor and Carer Needs Assessment Project. ${ }^{17}$

Although being a caregiver can be rewarding, our results confirm that the personal and financial consequences are often great. Our results add to existing literature, which describe the considerable burden and impacts experienced by caregivers of survivors of stroke. ${ }^{8,13,24}$ Our results confirm that the domains, most impacted on for caregivers, were those related to leisure activities and relationships. This may be explained by much of the assistance being provided during the caregiver's leisure and family time. ${ }^{7,12}$ Relinquishing leisure time activities and relationships with friends and family to be a caregiver can lead to feelings of social isolation, psychological strain, anxiety, and reduced quality of life. ${ }^{9,11,23}$ This can be compounded by increased financial strain due to the caregivers reduced ability to engage in paid work and the increased personal expenses associated with the survivors' disability needs. Practical solutions are needed to address these issues. Access to external support, adequate respite care, and financial assistance have been identified by caregivers as important mechanisms for allowing them to maintain previous activities and manage their caregiver role. ${ }^{12,15}$ It is concerning then, that almost one-quarter of those surveyed in our study, who felt that they needed these services, were not able access them.

Our results also add to a growing body of literature describing the interrelationships between survivor and caregiver characteristics and impacts when assessing caregiver burden or impacts. ${ }^{8,11,20-22,25}$ Changes in roles and interpersonal relationships between caregivers and survivors are yet another important aspect of caring, that many caregivers struggle with. ${ }^{13,23}$ This is highlighted by the strong interrelationship between spousal relationship impacts shown in our results at a median of 2 years following stroke.

Despite the large body of literature describing what is needed to minimize the impact on caregivers of survivors of stroke, there is little evidence on effective interventions to reduce caregiver impacts. Most interventions aimed at reducing caregiver burden or impacts have focused on structured caregiver training, rehabilitation-based interventions and information, support groups, and respite care in the early period following stroke. ${ }^{26-33}$ One small study, involving basic nursing and personal care techniques that also included a component specific to the patient and caregiver needs, significantly reduced caregiver burdens in the intervention group. ${ }^{27}$ However, a subsequent, larger, multicenter trial of the same intervention found no difference between the intervention and control group. ${ }^{30}$ The timing of the intervention (inpatient period) and time-limited nature of the intervention may have contributed to its lack of effectiveness in the long-term. Our results obtained from caregivers and survivors at 12 or more months following stroke suggest that effective interventions will require complex approaches directed at both caregivers and survivors that are personalized and responsive to the changing physical, emotional, and relationship needs of both survivors and caregivers. ${ }^{34}$ This should involve a combination of practical solutions, eg, appropriate and accessible respite care or financial support, counseling and psychological support, and ongoing access to quality resources and services.

Limitations of our study are consistent with the nature of cross-sectional surveys. It is likely that selection bias is present, especially with regard to those recruited through voluntary agencies such as the NSF. However, it is unclear as to whether this would produce an overestimation or an underestimation of the results. Also, characteristics between responders and nonresponders recruited through AuSCR for the survivor component of the survey were similar, indicating that responder bias is likely to be low. It was not possible to calculate a response rate for the caregiver component of the survey as we did not know what proportion of the total survivor sample $(n=765)$ had a caregiver. Temporal relationships between survivor unmet needs and caregiver impacts could not be established, ie, it is not possible to tell if having needs that are not being addressed caused caregiver impacts or whether caregivers who were experiencing impacts were less likely to be able to meet survivor needs. Finally, health systems and family structures are complex and differ between countries and cultures, and this may impact on the generalizability of our findings. However, our results are likely to represent situations in other, similar developed countries.

\section{Conclusion}

We provide new information on the association between survivor unmet needs and caregiver impacts at a median of 2 years following stroke. We have demonstrated a strong 
relationship between the extent to which survivor longterm needs are met and the degree to which caregivers are impacted across multiple aspects of their lives. We have also shown that caregivers are still experiencing large impacts as a consequence of taking on a caregiver role, at 12 or more months following stroke. Understanding the relationships between survivor needs, survivor problems, and caregiver impacts is important for the development of comprehensive and tailored interventions that can successfully support both caregivers and survivors living in the community. In particular, ongoing psychological support is needed to assist both the caregiver and survivor adapt to changes in their situations and the associated changes in their relationship. Further work is needed, involving strong consultation with consumers, health professionals, and health service managers, to better understand how community-based health professionals and services can best address this gap in providing long-term resources for caregivers of survivors of stroke.

\section{Acknowledgments}

The Stroke Survivor and Carer Needs Survey was commissioned by the National Stroke Foundation of Australia (NSF). We acknowledge the NSF staff members who were involved in survey design and production and contributed to data collection, participant recruitment, and report writing. We are also grateful to members of the Advisory Committee and the United Kingdom researchers who provided early input into the survey development, and staff from the Florey Institute of Neurosciences and Mental Health, who undertook data processing and teleform development and survey design.

We acknowledge staff from the following hospitals who assisted with recruitment and preparing ethics applications: Westmead Hospital, Fairfield Hospital, Belmont District Hospital, Orange Base Hospital, Coffs Harbour Base Hospital, Albury Wodonga Health Service - Albury Campus, Northern Hospital, Swan Hill District Hospital, Bairnsdale Hospital, Geelong Hospital, Hamilton Hospital, Prince Charles Hospital, The Queen Elizabeth Hospital, Flinders Medical Centre, Whyalla Hospital, Royal Hobart Hospital, Launceston General Hospital, Geraldton Hospital, and Alice Springs Hospital.

\section{Disclosure}

This project was commissioned and funded by the National Stroke Foundation. The Trust Company contributed funds via a grant to the NSF. Dominique Cadilhac is supported by a National Health and Medical Research Council (NHMRC)/ National Heart Foundation Research Fellowship (1063761) and Nadine Andrew is supported by an NHMRC Early Career Fellowship (1072053). The authors report no other conflicts of interest in this work.

\section{References}

1. Australian Institute of Health and Welfare. Stroke and Its Management in Australia: An Update. Australian Institute of Health and Welfare: ACT, Australia; 2013:159.

2. Hare R, Rogers $\mathrm{H}$, Lester $\mathrm{H}$, et al. What do stroke patients and carers want from community services? Fam Pract. 2006;23:131-136.

3. Sumathipala K, Radcliffe E, Sadler E, et al. Identifying the long-term needs of stroke survivors using the international classification of functioning, disability and health. Chronic Illness. 2011;8:31-44.

4. White J, Magin P, Attia J, et al. Exploring poststroke mood changes in community-dwelling stroke survivors: a qualitative study. Arch Phys Med Rehabil. 2008;89:1701-1707.

5. Ch'ng A, French D, McLean N. Coping with the challenges of recovery from stroke. J Health Psychol. 2008;13:1136-1146.

6. Turner B, Fleming J, Ownsworth T, et al. Perceived service and support needs during transition from hospital to home following acquired brain injury. Disabil Rehabil. 2011;33:818-829.

7. Dewey HM, Thrift AG, Mihalopoulos C, et al. Informal care for stroke survivors: results from the North East Melbourne Stroke Incidence Study (NEMESIS). Stroke. 2002;33:1028-1033.

8. Tooth L, McKenna K, Barnett A, et al. Caregiver burden, time spent caring and health status in the first 12 months following stroke. Brain Injury. 2005;19:963-974.

9. McCullagh E, Brigstocke G, Donaldson N, et al. Determinants of caregiving burden and quality of life in caregivers of stroke patients. Stroke. 2005;36:2181-2186.

10. Denno MS, Gillard PJ, Graham GD, et al. Anxiety and depression associated with caregiver burden in caregivers of stroke survivors with spasticity. Arch Phys Med Rehabil. 2013;94:1731-1736.

11. Cumming T, Cadilhac D, Rubin G, et al. Psychological distress and social support in informal caregivers of stroke survivors. Brain Impairment. 2008;9:152-160.

12. El Masry Y, Mullan B, Hackett M. Psychosocial experiences and needs of Australian caregivers of people with stroke: prognosis messages, caregiver resilience, and relationships. Top Stroke Rehabil. 2013;20: 356-368.

13. Perry L, Middleton S. An investigation of family carers' needs following stroke survivors' discharge from acute hospital care in Australia. Disabil Rehabil. 2011;33:1809-1900.

14. Ski C, O'Connell B. Stroke: the increasing complexity of carer needs. BJNN. 2007;39:172-179.

15. O'Connell B, Baker L. Managing as carers of stroke survivors: strategies from the field. IJNP. 2004;10:121-126.

16. National Stroke Foundation. Walk in Our Shoes. Stroke Survivors and Carers Report on Support after Stroke. Melbourne, Australia: National Stroke Foundation; 2007.

17. Andrew N, Kilkenny M, Naylor R, et al. Understanding long-term unmet needs in Australian survivors of stroke. Int J Stroke. 2014;9: 106-112.

18. McKevitt C, Fudge N, Redfern J, et al. Self-reported long-term needs after stroke. Stroke. 2011;42:1398-1403.

19. Belsley DA, Kuh E, Welsch RE. Regression Diagnostics. New York, NY: Wiley \& Sons; 1980.

20. Jönsson A-C, Lindgren I, Hallström B, et al. Determinants of quality of life in stroke survivors and their informal caregivers. Stroke. 2005; 36:803-808.

21. van Exela N, Koopmanschapa M, van den Bergc B, et al. Burden of informal caregiving for stroke patients. Cerebrovasc Dis. 2005;19:11-17.

22. Choi-Kwon S, Kim H-S, Kwon S, et al. Factors affecting the burden on caregivers of stroke survivors in South Korea. Arch Phys Med Rehabil. 2005;86:1043-1048. 
23. McPherson CJ, Wilson KG, Chyurlia L, et al. The caregiving relationship and quality of life among partners of stroke survivors: a crosssectional study. Health Qual Life Outcomes. 2011;9:29.

24. O'Shea R, Goode D. Effects of stroke on informal carers. Nurs Stand. 2013;28:43-47.

25. Smeets SM, van Heugten CM, Geboers JF, et al. Respite care after acquired brain injury: the well-being of caregivers and patients. Arch Phys Med Rehabil. 2012;93:834-841.

26. Legg L, Quinn T, Mahmood F, et al. Non-pharmacological interventions for caregivers of stroke survivors. Cochrane Database Syst Rev. 2011;10:CD008179.

27. Kalra L, Evans A, Perez I, et al. Training carers of stroke patients: randomised controlled trial. BMJ. 2004;328:1-5.

28. Mant J, Carter J, Wade D, et al. Family support for stroke: a randomised controlled trial. Lancet. 2000;356:808-813.

29. Hartke RJ, King RB. Telephone group intervention for older stroke caregivers. Top Stroke Rehabil. 2003;9:65-81.
30. Forster A, Dickerson J, Young J, et al. A structured training programme for caregivers of inpatients after stroke (TRACS): a cluster randomised controlled trial and cost-effectiveness analysis. Lancet. 2013;382:2069-2076.

31. Shyu YI, Chen MC, Chen ST, et al. A family caregiver-oriented discharge planning program for older stroke patients and their family caregivers. J Clin Nurs. 2008; 17:2497-2508.

32. Karahan AY, Kucuksen S, Yilmaz H, et al. Effects of rehabilitation services on anxiety, depression, care-giving burden and perceived social support of stroke caregivers. Acta Medica (Hradec Kralove). 2014;57:68-72.

33. Grasel E, Schmidt R, Biehler J, et al. Long-term effects of the intensification of the transition between inpatient neurological rehabilitation and home care of stroke patients. Clin Rehabil. 2006;20:577-583.

34. Tsai P-C, Yip P-K, Tai JJ, et al. Needs of family caregivers of stroke patients: a longitudinal study of caregivers' perspectives. Patient Prefer Adherence. 2015;9:449-457.

\section{Publish your work in this journal}

Patient Preference and Adherence is an international, peer-reviewed, open access journal that focuses on the growing importance of patient preference and adherence throughout the therapeutic continuum. Patient satisfaction, acceptability, quality of life, compliance, persistence and their role in developing new therapeutic modalities and compounds to optimize clinical outcomes for existing disease states are major areas of interest for the journal. This journal has been accepted for indexing on PubMed Central. The manuscript management system is completely online and includes a very quick and fair peer-review system, which is all easy to use. Visit http://www. dovepress.com/testimonials.php to read real quotes from published authors.

Submit your manuscript here: http://www.dovepress.com/patient-preference-and-adherence-journal 